\title{
Noninvasive ventilation for pediatric interfacility transports: a retrospective study
}

\author{
Samantha Holbird ${ }^{1} \cdot$ Tanya Holt $^{2} \cdot$ Adam Shaw $^{2} \cdot$ Gregory Hansen $^{2}$
}

Received: 20 December 2019 / Accepted: 31 March 2020 / Published online: 13 May 2020

(c) Children's Hospital, Zhejiang University School of Medicine 2020

\begin{abstract}
Background To characterize pediatric patients supported with continuous positive airway pressure and bilevel positive airway pressure (CPAP/BiPAP) or high-flow nasal cannula (HFNC) during interfacility transport (IFT).

Methods A retrospective study with a provincial pediatric transport team from a tertiary hospital pediatric intensive care unit. Pediatric patients aged 28 days to $<17$ years, who required IFT between January 2017 and December 2018, were identified through a transport registry and were included in the study.

Results A total of 118 (26.7\%) patients received CPAP/BIPAP or HFNC support for IFT. The most common respiratory diagnosis was bronchiolitis (46\%). These patients were placed on respiratory support, 31.4 minutes after the transport team's arrival. None required intubation during their IFT, despite mean transport times of 163 minutes.

Conclusions This study may provide important information for programs with large catchment areas, in which large distances and transport times should not be barriers to NIV implementation.
\end{abstract}

Keywords Children $\cdot$ Critical illness $\cdot$ Intubation $\cdot$ Noninvasive ventilation $\cdot$ Transportation of patients

\section{Introduction}

With critically ill neonates and children in acute respiratory distress, conventional mechanical ventilation (CMV) has frequently been employed during interfacility transports (IFTs). With advancements in noninvasive technologies that mitigate some of the risks and complications associated with $\mathrm{CMV}$, an increased interest and utilization have been documented $[1,2]$.

Noninvasive ventilation (NIV) - in the form of continuous positive airway pressure (CPAP) and bilevel positive airway pressure (BiPAP) — was first reported in adult IFTs for chronic obstructive pulmonary disease, acute cardiogenic pulmonary edema, and pneumonia [3]. In pediatrics, NIV in the pediatric intensive care unit (PICU) first demonstrated improved vital signs and decreased both mortality and need

Gregory Hansen

gregory.hansen@usask.ca

1 College of Medicine, University of Saskatchewan, Saskatoon, SK, Canada

2 Division of Pediatric Critical Care, Jim Pattison Children's Hospital, 103 Hospital Drive, Saskatoon, SK S7N 0W8, Canada for mechanical ventilation $[2,4,5,6]$. Pediatric NIV during IFTs would follow [4] by mimicking its increased utilization in the PICU $[3,7,8]$.

A recent systematic review suggested that NIV is safe and feasible in the transport environment [9], but mortality, intubation rates, and effect on length of stay have not been fully established. The objectives of this retrospective chart review study were to characterize pediatric patients requiring IFTs and NIV and to examine their clinical outcomes, including the need for airway instrumentation.

\section{Methods}

\section{Provincial pediatric transport program}

In Saskatchewan, over 450 patients per year are transported from referring centers to the Royal University Hospital in Saskatoon. The province offers unique challenges including inclement weather and vast geography $\left(651,900 \mathrm{~km}^{2}\right)$ with a population of nearly 1.2 million. Pediatric intensivists, who provide medical oversight and triage for all IFT missions, have trained the specialized pediatric transport team to provide advanced treatment 
including airway instrumentation, chest tubes, and emergency cricothyrotomies.

Utilization of NIV and high-flow nasal cannula (HFNC) on IFTs began in the year 2014. NIV was provided via large bore cannula, total face mask, or full-face mask depending on patient size and compliance, and HFNC is provided via the Optiflow Jr. interface. The device used for CPAP and BiPAP was the Hamilton T1 transport ventilator with NIV and NIV-ST modes.

A trial of HFNC, as we have previously described [10], is undertaken at the referring center after ruling out immediate need for intubation and balancing variables such as time to tertiary care, road versus air transport, and diagnosis trajectory. NIV was typically not initiated by referring centers owing to their lack of equipment and/or in-house respiratory therapy support. At present, a protocol for NIV has not been established for our IFTs. Instead, the decision to proceed with NIV and preliminary settings was made following discussion with the pediatric intensivist and transport team and following appropriate observation of respiratory symptom improvement. In addition, an approach to escalation of settings during transport was discussed, with the most common triggers being increased oxygen requirements or work of breathing. Blood gas analyses were routinely performed prior to initiation of NIV or HFNC, but end tidal $\mathrm{CO}_{2}$ was not, because interface leaks led to unreliable trends or to inability to measure with certain modes.

\section{Chart review}

Pediatric patients who required IFT between January 2017 and December 2018 were identified through the provincial pediatric transport program's registry. All children aged 30 days to $<17$ years who were subsequently admitted to the PICU were included in the study. Children with a prior advanced care directive or tracheostomy were excluded. Baseline patient demographics and transport metrics included season of transport, distance and time of return leg (transport team departure to PICU admission), transport team assessment, Transport Risk Assessment in Pediatrics (TRAP) score [11], PICU arrival TRAP score, mode of ventilatory support, highest ventilatory support, highest fraction of inhaled oxygen $\left(\mathrm{FiO}_{2}\right)$, and need for sedation. Data on HFNC were also collected but analyzed separately from NIV. TRAP is an objective assessment tool in which an elevated score is associated with a PICU admission for more than 24 hours [11]. Mode of transport (ground or fixed wing) was determined. Lastly, transport and patient outcome measures were collected and recorded. The University of Saskatchewan Biomedical Research Ethic Board approved the study (Bio-REB 921).

\section{Statistical analysis}

Discrete variables were reported as percentages, and continuous data as means and standard deviations (SD). Proportions were compared using a two-tailed Fisher's exact test. Difference in means and their 95\% confidence intervals (CI) were calculated. Significance level was considered at $P=0.05$. STATA 14 software (StataCorp. 2015. Stata Statistical Software: Release 14. College Station, TX: StataCorp LP) was utilized for all analyses.

\section{Results}

A total of 120 patients (Table 1) on NIV or HFNC for IFT were studied (26.7\% of transport cohort), but 2 had incomplete records and were excluded from analysis. The most common respiratory diagnosis was bronchiolitis $(n=54$, $45.8 \%$ ). IFT metrics are also summarized in Table 1. Differences of mean TRAP scores between transport team arrival and tertiary center handover were significant $(95 \% \mathrm{CI}-0.90$ to $-0.04, P=0.03$ ).

Respiratory support during IFT is summarized in Table 2. Eleven patients were already on NIV or HFNC before the

Table 1 Patient demographics $(n=118)$ and interfacility transport metrics

\begin{tabular}{ll}
\hline Variables & Value \\
\hline Age (y), mean (SD) & $2.3(3.6)$ \\
Gender (female), $n(\%)$ & $50(42)$ \\
Weight (kg), mean (SD) & $12.9(12.5)$ \\
Diagnosis, $n(\%)$ & \\
Bronchiolitis & $54(46)$ \\
Asthma & $15(13)$ \\
Pneumonia & $27(23)$ \\
Others & $22(19)$ \\
Transport mode, $n(\%)$ & \\
Ground & $48(41)$ \\
Fixed wing & $70(59)$ \\
Season of transport, $n(\%)$ & \\
Spring & $27(23)$ \\
Summer & $18(15)$ \\
Autumn & $28(24)$ \\
Winter & $45(38)$ \\
Distance (km), mean (SD) & $236(105)$ \\
Transport time (min), mean (SD) & $163(51)$ \\
TRAP scores, mean (SD) & \\
Transport team arrival & $4.6(1.8)$ \\
Tertiary center handover & $4.1(1.5)$ \\
\hline
\end{tabular}

TRAP transport risk assessment in pediatrics, $S D$ standard deviation 
Table 2 Respiratory support during interfacility transport

\begin{tabular}{ll}
\hline Variables & Value \\
\hline HFNC, $n(\%)$ & $9(8)$ \\
Bronchiolitis diagnosis, $n(\%)$ & $6(67)$ \\
Initial TRAP score, mean (SD) & $3.7(1.3)$ \\
CPAP, $n(\%)$ & $40(34)$ \\
Bronchiolitis diagnosis, $n(\%)$ & $27(68)$ \\
Initial TRAP score, mean (SD) & $4.0(0.8)$ \\
BiPAP, $n(\%)$ & $69(59)$ \\
Bronchiolitis diagnosis, $n(\%)$ & $38(55)$ \\
Initial TRAP scoree, mean (SD) & $6.1(1.6)$ \\
Maximum support, $n(\%)$ & \\
HFNC $\left(\mathrm{L} / \mathrm{kg}_{\mathrm{m}}\right.$ min) & $2(8)$ \\
CPAP $\left(\mathrm{cm} \mathrm{H}_{2} 0\right)$ & $10(34)$ \\
BiPAP $\left(\mathrm{cm} \mathrm{H}_{2} 0\right)$ & $20 / 10(59)$ \\
In-flight intubations, $n$ & 0 \\
Fraction of inspired oxygen, mean (SD) & $0.43(0.20)$ \\
Sedation required ${ }^{\mathrm{a}}, n(\%)$ & $4(3)$ \\
\hline
\end{tabular}

$H F N C$ high-flow nasal cannula, CPAP continuous positive airway pressure, $B i P A P$ bilevel positive airway pressure, $S D$ standard deviation. ${ }^{\mathrm{a}}$ Midazolam $0.05 \mathrm{mg} / \mathrm{kg}$ by intravenous injection

team arrived; the remaining patients were placed on respiratory support, 31.4 minutes (SD 23.17) after the transport team's arrival. No patients required intubation during IFT, but three patients $(2.5 \%)$ experienced desaturations $<80 \%$ due to apneas.

Outcomes, including maximal respiratory support, duration of support, length of stay, and mortality are summarized in Table 3. Of note, $16.1 \%(n=19)$ required conventional mechanical ventilation and were intubated an average of 57.6 (SD 33.4, range 0-528) hours after admission. One patient $(0.8 \%)$ died due to complications associated with acute respiratory distress syndrome.

\section{Discussion}

This study provides a synopsis of the use of NIV and HFNC during pediatric IFTs and their outcomes by a provincial pediatric transport program. Our primary observations suggest that its utilization is safe and does not lead to untimely intubations.

This review's results are similar to those described in previously published literature and suggest that NIV is safe for IFTs $[12,13]$. The only negative outcomes were significant hypoxemia in three patients that were resolved with stimulation or with bag valve mask ventilation. There was also one case of bag valve mask use which was required due to an equipment issue, but NIV was replaced without incident once the issue was resolved. An overall improvement of
Table 3 Clinical and respiratory support outcomes

\begin{tabular}{ll}
\hline Variables & Value \\
\hline Duration of NIV support (d), mean (SD) & $2.7(3.4)$ \\
Duration of HFNC support (d), mean (SD) & $1.2(2.4)$ \\
Conventional mechanical ventilation & \\
Required CMV, $n(\%)$ & $19(16)$ \\
Time of intubation after admission (h), mean (SD) & $58(33)$ \\
Duration of CMV (d), mean (SD) & $8.0(1.4)$ \\
Pneumonia diagnosis, mean (SD) & $8(42)$ \\
Initial TRAP score, mean (SD) & $5.2(1.6)$ \\
HFNC during transport, $n(\%)$ & $1(5)$ \\
CPAP during transport, $n(\%)$ & $7(37)$ \\
BiPAP during transport, $n(\%)$ & $11(58)$ \\
PICU admission (d), mean (SD) & $6.0(7.3)$ \\
Hospital admission (d), mean (SD) & $8.4(10.7)$ \\
Mortality, $n(\%)$ & $1(1)$ \\
30-day readmission rate, $n(\%)$ & $8(7)$ \\
\hline
\end{tabular}

NIV noninvasive ventilation, $H F N C$ high-flow nasal cannula, $C M V$ continuous mechanical ventilation, $C P A P$ continuous positive airway pressure, $B i P A P$ bilevel positive airway pressure, $P I C U$ pediatric intensive care unit, $S D$ standard deviation

TRAP scores during transport suggests that vital signs and clinical status are grossly stabilized. Together, these results highlight the importance of an experienced transport team capable of appropriately selecting patients and promptly recognizing and responding to complications. It is also prudent to note that none of our patients required conversion to CMV during IFT, despite long transport times and distances.

Higher than previously reported [14], $16 \%$ of patients eventually required CMV in the PICU. Although this percentage may seem large, patients typically were intubated later (mean 57 hours post PICU admission) and required longer ventilator times (mean 8 days), which perhaps suggests disease progression rather than a failure of NIV. At the very least, questions of what percentages are acceptable need to be considered. With a more aggressive strategy of instrumenting airways be employed, several patients could have been unnecessarily supported with CMV and exposed to its associated risks, such as ventilator-associated pneumonia, airway injury, and sedation. A recent Canadian study reported prehospital and PICU NIV use in bronchiolitis and demonstrated similar outcomes with less complications and with fewer interventions than patients receiving CMV [14]. Surely a balance exists in providing patients safe opportunities to declare clinical trajectory on NIV support, but this has not yet been clearly elucidated.

Finally, our study also may have captured a growing and unique patient cohort in pediatric intensive care: patients that cannot be supported by low-flow nasal cannula, but require a short duration of ventilatory support. The majority of our patients were supported for less than 3 days of 
NIV and $>1$ day of HFNC despite respiratory compromise in referring centers. Because bronchiolitis and asthma diagnoses accounted for nearly $60 \%$ of our cohort, short duration positive pressure for lung recruitment or maintenance of lower airway patency could have been responsible for these rapid turnarounds. Furthermore, this cohort is particularly important because a certain percentage would certainly have been intubated in the past. Our crude IFT data showed that in $2019,62 \%$ of patients requiring respiratory support were initiated on NIV; in 2014, it was less than $10 \%$.

There are a few limitations to our study. First, the retrospective nature of the study is a major limitation because the data depend on accurate recording. However, significant events during IFTs should have been captured by overlapping quality control processes, thereby mitigating the potential of missing serious adverse outcomes. Second, the lack of a protocol for NIV management during IFTs should be stated. This is an excellent area for ongoing research that we are currently pursuing. Finally, interpretation of our results should be done by considering that we did not utilize a historical cohort control in our methodology.

NIV and HFNC are common during pediatric IFTs especially for bronchiolitis. After our specialized transport team initiated NIV or HFNC, escalation to CMV was not necessary during the IFT, despite mean transport times of 2.5 hours. This retrospective study may suggest the utility of an NIV trial in suitable patients, so that instrumentation of the airway and CMV are avoided whenever possible.

Author contributions GH conceptualized the study and analyzed the data. TH co-conceptualized the study and interpreted the data. AS acquired the data from the transport registry. SH acquired the data from the hospital charts and wrote the initial manuscript. All authors revised and approved the final manuscript.

Funding None.

\section{Compliance with ethical standards}

Ethical approval Ethical approval granted by the University of Saskatchewan (Bio-REB 921).

Conflict of interest No financial or non-financial benefits have been received or will be received from any party related directly or indirectly to the subject of this article.

\section{References}

1. Baird SJ, Spiegelman JB, Prianti R, Frudak S, Schleien CL. Noninvasive ventilation during pediatric interhospital ground transport. Prehosp Emerg Care. 2009;13:198-202.

2. Wolfler A, Calderini E, Iannella E, Conti G, Biban P, Dolcini A, et al. Evolution of noninvasive mechanical ventilation use: a cohort study among Italian PICUs. Pediatr Crit Care Med. 2015;16:418-27.

3. Roessler MS, Schmid DS, Michels P, Schmid O, Jung K, Stöber $\mathrm{J}$, et al. Early out-of-hospital non-invasive ventilation is superior to standard medical treatment in patients with acute respiratory failure: a pilot study. Emerg Med J. 2012;29:409-14.

4. Null D Jr, Crezee K, Bleak T. Noninvasive respiratory support during transportation. Clin Perinatol. 2016;43:741-54.

5. Franklin D, Fraser JF, Schibler A. Respiratory support for infants with bronchiolitis, a narrative review of the literature. Pediatr Respir Rev. 2019;30:16-24.

6. Morris JV, Ramnarayan P, Parslow RC, Fleming SJ. Outcomes for children receiving noninvasive ventilation as the firstline mode of mechanical ventilation at intensive care admission: a propensity score-matched cohort study. Crit Care Med. 2017;45:1045-53.

7. Thompson J, Petrie DA, Ackroyd-Stolarz S, Bardua DJ. Out-ofhospital continuous positive airway pressure ventilation versus usual care in acute respiratory failure: a randomized controlled trial. Ann Emerg Med. 2008;52:232-41.

8. Mal S, McLeod S, Iansavichene A, Dukelow A, Lewell M. Effect of out-of-hospital noninvasive positive-pressure support ventilation in adult patients with severe respiratory distress: a systematic review and meta-analysis. Ann Emerg Med. 2014;63:600-7.

9. Cheema B, Welzel T, Rossouw B. Noninvasive ventilation during pediatric and neonatal critical care transport: a systematic review. Pediatr Crit Care Med. 2019;20:9-18.

10. Hansen G, Hochman J, Garner M, Dmytrowich J, Holt T. Pediatric early warning score and deteriorating ward patients on high-flow therapy. Pediatr Int. 2019;61:278-83.

11. Kandil S, Sanford HA, Northrup V, Bigham M, Giuliano JS Jr. Transport disposition using the transport risk assessment in pediatrics (TRAP) score. Prehosp Emerg Care. 2012;16:366-73.

12. Millán N, Alejandre C, Martinez-Planas A, Cartig J, Esteban E, Pons-Òdena M. Noninvasive respiratory support during pediatric ground transport: implementation of a safe and feasible procedure. Respir Care. 2017;62:558-65.

13. Schlapbach L, Schaefer J, Brady A, Mayfield S, Schibler A. Highflow nasal cannula (HFNC) support in interhospital transport of critically ill children. Intensive Care Med. 2014;40:592-9.

14. Essouri S, Baudin F, Chevret L, Vincent V, Emeriaud G, Jouvet P. Variability of care in infants with severe bronchiolitis: lessinvasive respiratory management leads to similar outcomes. J Pediatr. 2017;188:156-63.

Publisher's Note Springer Nature remains neutral with regard to jurisdictional claims in published maps and institutional affiliations. 